\title{
Esquemas de organización espacial para bases de datos de redes de conocimiento. Alternativas para representar sus elementos y relaciones en tiempo real
} \author{
in real time \\ - María Elena Tosello \\ Universidad Nacional del Litoral, Argentina \\ mtosello@fadu.unl.edu.ar \\ - Guillermo Luján Rodríguez \\ Universidad Nacional de Rosario, Argentina \\ guille@fceia.unr.edu.ar
}

Spatial organization schemes for databases of knowledge networks. Alternatives to represent its elements and relationships

\begin{abstract}
The capability to observe the heterogeneous elements of knowledge networks in virtual environments, and the dynamics of these performative spaces, allows evaluating and reflecting on the quality of interactions in construction and recognition mediated processes. This paper presents the first results of an innovative proposal of visualization that integrates design methodologies and is based on an ad-hoc software for taking real-time information. As corollary, it debates about the relationship between representation, design and conception of space in a complex and augmented sociotechnical context, configured and experimented as a web of events that interacts at multiple levels of articulation.
\end{abstract}

Keywords: Database, Representation, Interface-Space, Knowledge Networks, Mediated Processes

\section{Introducción}

El espacio actual, aumentado y simbiótico, está configurado por ámbitos físicos y virtuales interdependientes, y si bien los espacios virtuales interactivos o espacios-interfaz son lugares para la imaginación y la creación colaborativa, cabe recordar que el hombre es un "ser en situación" (Heidegger, 1954), que no percibimos un mundo objetivo, sino que "vivimos" el mundo en un espacio propio que se genera al actuar y que no puede separarse de nuestra propia historia de acciones, tanto biológicas como sociales, como sostienen Maturana y Varela (1984) en "El árbol del conocimiento".

Cada sujeto percibe el mundo desde el espacio visual propio, relacionando los nuevos aprendizajes con experiencias y conocimientos previos, traduciendo a su manera lo que percibe y generando nuevos sentidos e interpretaciones (Rancière, 2010) a partir de un conocimiento vivido mediante experiencias subjetivas e intersubjetivas. Entonces ¿cómo visibilizar a los sujetos, sus producciones y relaciones en un espacio virtual comunitario?

Disponer de la capacidad para observar los elementos heterogéneos (sujetos, acciones, medios, aplicaciones, etc.) de las redes de conocimiento en el entorno virtual, y las dinámicas performativas propias de estos espacios, permitirá indagar y reflexionar sobre la calidad de las interacciones que tienen lugar en procesos de construcción y reconocimiento participativos, con diferentes grados de mediatización. Esta reflexión posibilitará la apropiación responsable de los conocimientos por parte de las diversas comunidades, desde sus propios contextos, prácticas y valores. En este sentido, es fundamental la visibilidad de los procesos que se manifiestan en las relaciones intersubjetivas, las actividades, las herramientas y los recursos utilizados, las producciones, los roles y perfiles de los sujetos participantes.

En la década del '90, Lévy y Authier (1992) desarrollaron unaherramientapara representar losconocimientoscolectivos a través de "árboles de conocimientos", que hacían visible al individuo en el espacio del saber con todos los conocimientos que acumuló en su paso por el sistema educacional formal, las tradiciones de su familia y los avatares de su vida, y que le daban identidad en los mundos de significación que exploró y contribuyó a crear. El saber individual se representaba con un "emblema": la imagen del trayecto o aprendizaje individual en el "árbol del conocimiento" de una comunidad.

Pero las concepciones y configuraciones del espacio son múltiples, implican diversas interpretaciones del mundo y sugieren múltiples modos de habitar, y así, diferentes espacialidades inducirán distintos comportamientos (Doberti, 1998). A pesar de que tanto Maturana y Varela como Lévy y Authier, se valen de la imagen de un árbol para representar el conocimiento, Alexander (1965) propone el modelo conceptual de la trama o la red, la estructura organizacional 
de las ciudades antiguas, de las cosas vivas, de las grandes sinfonías, con su riqueza formal y sus variadas secuencias. La superposición y multiplicidad de la red no son menos ordenadas que la del rígido árbol sino más, son los atributos de una configuración más densa, robusta, sutil y compleja.

El objetivo de este trabajo es investigar alternativas de representación de los datos generados por redes socio-técnicas de conocimiento, para facilitar la lectura, interpretación y resignificación de la información.

\section{Metodología}

Los elementos heterogéneos de un entorno virtual interactivo que participan en procesos de producción y reconocimiento colaborativos, se agrupan en bases de datos -BD- que siempre están creciendo. Estos elementos se pueden definir en función a las siguientes variables: tipo de objeto; nombre; fecha; tema; descripción; edad; relación; relevancia o rol; nivel de interacción, cantidad de visualizaciones, descargas o likes; tiempo de interacción o edición; o las que se consideren pertinentes en cada caso particular. Cada elemento se puede representar mediante signos con diversos grados de abstracción, desde íconos hasta formas geométricas abstractas. Para representar los elementos, se propone asociar las variables a coordenadas espaciales (relativas a los ejes $\mathrm{x}, \mathrm{y}$, z), y a las propiedades: forma, tamaño, color, textura y opacidad o transparencia, las cuales pueden cambiar en el tiempo. Así, cada elemento se representará con una combinación particular de ubicación, forma, color, etc.

Se evaluaron diversas combinaciones de propiedades, por ejemplo la utilización de diferentes formas para representar los distintos tipos de elementos, y que todos sean del mismo color; o usar una forma única para bajar el ruido visual, y el color para definir el tipo de signo. Más allá de que el lenguaje utilizado sea realista o abstracto, cualquier composición espacial puede ser puesta en una profundidad de campo simulada, distribuyendo los objetos en diferentes capas o layers de un espacio virtual en 3D (Manovich, 2013). Se dispusieron objetos con la misma forma y distintos colores en planos horizontales y andariveles o subtemas, configurando una estructura de distribución por capas temáticas que posteriormente se interrelacionarían, y se observó que en el caso de utilizar una sola forma, se debería colocar un ícono en su núcleo, para identificar el tipo de objeto.

Se verificó que la disposición vertical de las capas mejora la legibilidad de la información como también, que una grilla estructural (visible o no) le resta organicidad a la propuesta. Se estudiaron alternativas formales para los elementos, sus agrupamientos y transformaciones, las que pueden definirse mediante algoritmos. Se realizaron comparaciones entre formas (de las más geométricas a las más complejas) para observar cuanto resistían como unidades reconocibles. A medida que se conformaba el enjambre de objetos, se fueron descartando texturas y transparencias que en principio se incorporaron, ya que las texturas no son observables y con altos niveles de transparencia se produce una superposición visual que no permite reconocer los elementos.

\section{Resultados}

Se presenta un caso de aplicación que incluye las variables de una herramienta diseñada ad-hoc para el seguimiento y la evaluación de procesos de interactividad mediatizados por plataformas educativas, denominada SEPI-DHD (Rodríguez, 2011). SEPI analiza la interactividad en una doble dimensión:

En primera instancia considera las características de cada evento en concreto, integrando la información necesaria en función de las interacciones de los participantes, las cuales están definidas por la identificación del participante (id), el rol del mismo, el Paquete Hipermedial o curso en el cual participa, el tipo de $\mathrm{PH}$, la herramienta sobre la cual realiza la interacción, el tipo de herramienta, el servicio con el cual interactúa, y tiempo, día y hora de la interacción.

En segunda instancia, tiene en cuenta el proceso en su dinámica compleja, y por lo tanto el nivel de interactividad para un evento concreto dependerá de la integración de las variables y no sólo de esa interacción en particular. Así, el valor del nivel de interactividad calculado dependerá del devenir temporal hasta llegar al evento concreto.

La propuesta de SEPI-DHD se considera superadora en relación a los instrumentos estadísticos disponibles, donde no existe un modelo de interactividad subyacente que posibilite la interpretación procesual de los eventos, y por lo tanto, sólo permiten análisis cuantitativos. A su vez, la implementación de la evaluación ofrece mayores niveles de flexibilidad para los valores de los indicadores parciales y globales. Esto es factible dado que el responsable/s de la evaluación tiene la capacidad de ponderar los diversos coeficientes, subrayando aquel atributo que considere más importante en el proceso.

Como resultado del proceso de simulación se obtiene una tabla con sus valores separados por coma (extensión csv: comma-separated values). El vector de salida para cada participación contiene entre otros, el nombre del participante, su rol, el curso sobre el que realiza la interacción, la herramienta, el servicio, la fecha y hora, y por último, el nivel de interactividad calculado (Fig. 1). 


\begin{tabular}{|c|c|c|c|c|c|c|}
\hline ID Usuario & Rol & Paquete Hipermedial / Curso & Herramienta & Servicio & Fecha & Nivel de Interactividad \\
\hline Patricia San Martín & maintain & Niñ@s y ciudadanía & forums & revise & $04 / 05 / 201521$ & 1 \\
\hline Patricia San Martín & maintain & Niñ@s Expresiv@s & forums & revise & $04 / 05 / 201521$ & 1 \\
\hline Patricia San Martín & maintain & Red Social Cossettini & forums & revise & $04 / 05 / 201521$ & 1 \\
\hline Patricia San Martín & maintain & Diario de Maestr@ & forums & revise & $04 / 05 / 201521$ & 1 \\
\hline Marisa Lanteri & maintain & Diario de Maestr@ & forums & new & $09 / 05 / 201520$ & 1 \\
\hline Marisa Lanteri & maintain & Diario de Maestr@ & forums & read & $09 / 05 / 201520$ & \\
\hline Manuel Encina & maintain & Diario de Maestr@ & chat & read & $14 / 05 / 201519$ & 4 \\
\hline Javiera Diaz & access & Diario de Maestr@ & chat & read & $27 / 05 / 201514$ & 7 \\
\hline Patricia San Martín & maintain & Diario de Maestr@ & forums & revise & $29 / 05 / 201512$ & \\
\hline Patricia San Martín & maintain & Niñ@s Expresiv@s & forums & revise & $29 / 05 / 201512$ & 4 \\
\hline Patricia San Martín & maintain & Niñ@s Expresiv@s & forums & revise & $29 / 05 / 201512$ & 1 \\
\hline Patricia San Martín & maintain & Niñ@s Expresiv@s & forums & revise & $29 / 05 / 201512$ & 4 \\
\hline Patricia San Martín & maintain & Niñ@s y ciudadanía & forums & revise & $29 / 05 / 201512$ & 4 \\
\hline Florencia Dietrich & access & Niñ@s y ciudadanía & content & new & $03 / 06 / 201517$ & 1 \\
\hline Florencia Dietrich & access & Niñ@s y ciudadanía & content & new & $03 / 06 / 201517$ & 1 \\
\hline Florencia Dietrich & access & Niñ@s y ciudadanía & content & new & $03 / 06 / 201517$ & 7 \\
\hline
\end{tabular}

Figura 1: Parte de la tabla generada por SEPI- DHD.

En relación a la representación de los datos generados por SEPI para facilitar su interpretación y resignificación, luego de analizar aplicaciones disponibles que permiten la visualización de datos tales como Gephi, Many Eyes, Tableau, Google Fusion Tables, NodeXL, Weave, DataDriven Documents (D3 js), Dygraphs, etc., se desarrolló una forma de representación original. Se realizaron diversas pruebas explorando alternativas de relación entre las variables y las propiedades de ubicación, forma y color, enunciadas anteriormente.

Se propone un diseño a nivel conceptual en el cual se representa un proceso genérico de construcción de bases de datos colaborativas y sus relaciones en tiempo real. En el mismo, se optó por representar los distintos tipos de elementos (sujetos, producciones, actividades, etc.) con diferentes formas, donde el tamaño indica la relevancia del tema, la jerarquía del rol del participante, o simplemente el tamaño del archivo, y el color, el nivel de interacción, más frío es más pasivo (sólo lectura), más cálido es más activo (participación en foros, subida de archivos, etc.); o la cantidad de visualizaciones, descargas o likes, que pueden considerarse marcadores de popularidad o urgencia (destacado, importante, irrelevante). El nombre aparecería al pasar el mouse por encima del elemento, la descripción al hacer clic, y las relaciones entre producciones, sujetos, entre producción y aportante, o entre usuarios y herramientas, se materializan con líneas de diversos formatos (Fig. 2).

\section{Caso de aplicación}

Actualmente se está trabajando en la visualización de los procesos de interactividad realizados en el espacio-interfaz del "Archivo Pedagógico Cossettini", perteneciente al Instituto de Investigaciones en Ciencias de la Educación de Rosario, Argentina (IRICE: CONICET-UNR). La navegación a través del espacio virtual del Archivo, fue pensada a partir de cuatro claves de ingreso o tipos de itinerarios que fueron creados como cursos, en función de los materiales existentes en el archivo: Niñ@s y Ciudadanía, Diario de Maestr@, Red social Cossettini y Niñ@s expresiv@s.

Dentro de cada uno de estos itinerarios, existen diferentes recursos y actividades. La tecnología utilizada es SAKAI CLE (Collaborative Learning Enviroment) una plataforma educativa de código abierto programada en JAVA que corre sobre un servidor web. La aplicación SEPI-DHD -también programada en JAVA- funciona sobre el mismo servidor $\mathrm{y}$ consulta en tiempo real una base de datos MySQL donde se guarda la información.

La tabla que arroja SEPI (Fig. 1), es postprocesada para construir dos tipos de visualizaciones: una en el plano y otra en el espacio.

Visualización en el plano:

La Fig. 3 muestra todas las interacciones realizadas en el espacio-interfaz del Archivo Pedagógico Cossettini en un período de 13 semanas (desde el 4 de mayo al 31 de julio de 2015), a través de una visualización innovadora donde se asignaron las siguientes correspondencias:

- $\quad$ posición en $\mathrm{x}$ : tiempo (en semanas);

- posición en y: curso sobre el cual se realiza la interacción (Diario de maestr@ - Niñ@s Expresiv@s - Niñ@s y Ciudadanía - Red Social Cossettini);

- icono: herramienta (Forum, Chat, Content, Anuncio, Wiki);

- tamaño: nivel de interactividad de la participación, resultado de la evaluación procesual de los eventos mediante la aplicación SEPI-DHD;

- color: tipo de servicio (New, Response, Revise, Read, Delete), más cálido es más activo, más frío es más pasivo.

La forma final de cada itinerario es el resultado de la demarcación con curvas de bezier de las interacciones realizadas en cada semana, sobre la base de una trama uniforme de filas y columnas del mismo tamaño inicial, donde 


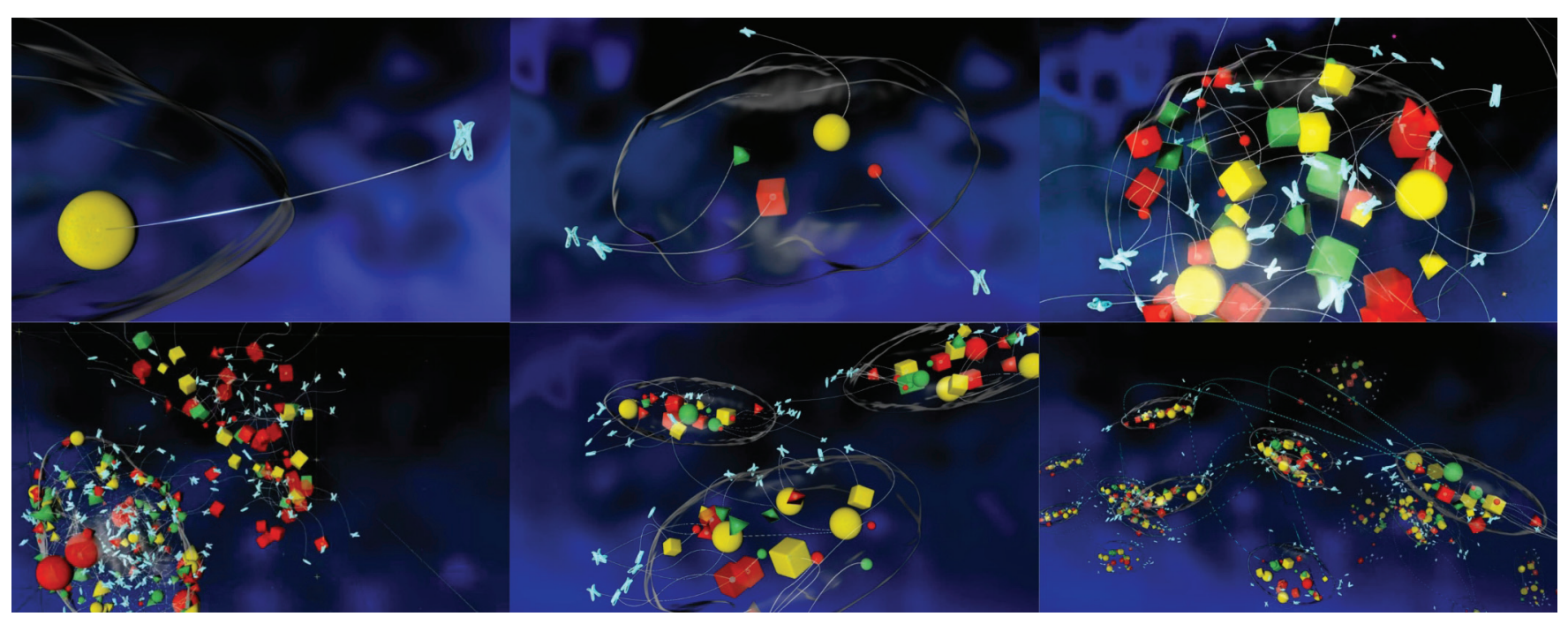

Figura 2: Representación del proceso de construcción colaborativa de una BD (Dorigo, 2014).

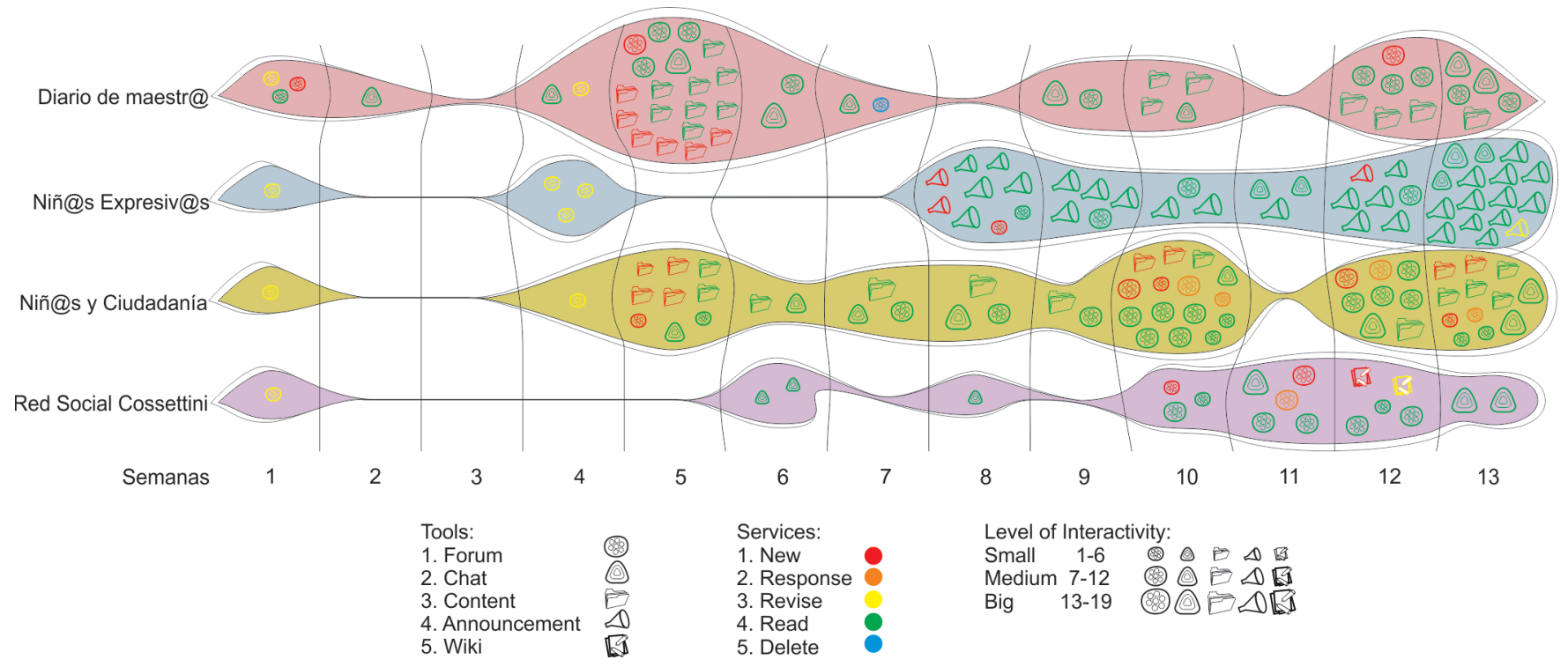

Figura 3: Representación de los procesos de interactividad realizados en el espacio-interfaz del "Archivo Pedagógico Cossettini".

cada celda se agranda o achica en función a la cantidad de eventos y el nivel de interactividad calculado.

Visualización en el espacio:

A una vista isométrica de la gráfica anterior cuyos eventos se agrupan en racimos de datos, se incorpora el eje z que se utiliza para representar las relaciones que cada sujeto estableció dentro de un mismo curso y entre distintos cursos, distribuidas en el tiempo.

La visualización espacial propuesta permite analizar e interpretar aspectos performativos de procesos de producción colectivos, interactivos y dinámicos desde una mirada integradora, a partir de la vinculación transversal de los distintos espacios curriculares. La Fig. 4 presenta los trayectos realizados por algunos de los participantes en el espacio-interfaz del Archivo Cossettini.

Este tipo de representación brinda información calificada para una evaluación cualitativa y en tiempo real sobre cómo se desarrollan las prácticas de participación responsable a través de redes socio-técnicas para educar, investigar, gestionar y producir en el actual contexto aumentado y simbiótico. 


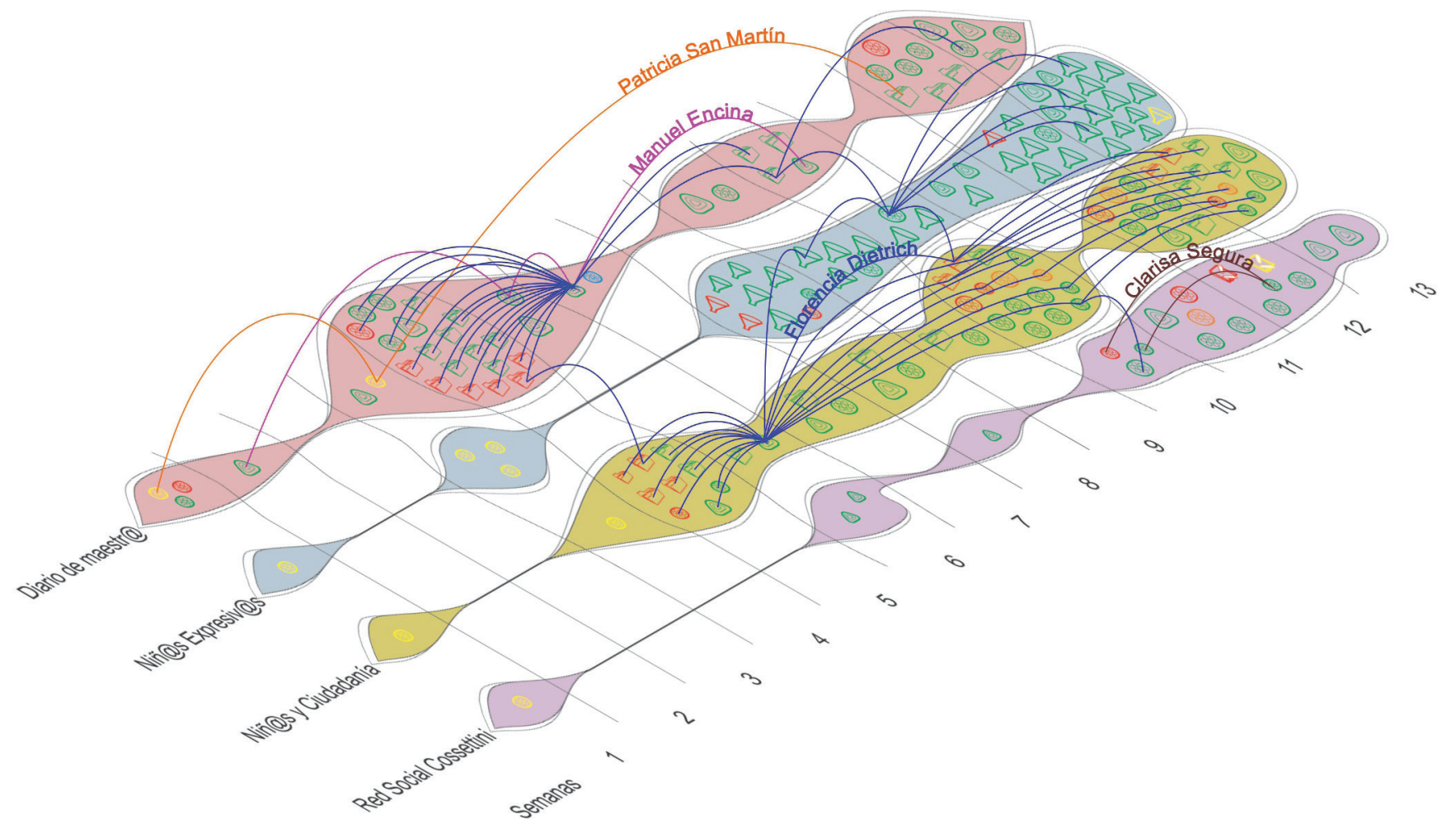

Figura 4: Representación de los trayectos de algunos participantes.

\section{Discusión}

Cada visualización de información implica un proceso complejo de construcción de sentido donde intervienen múltiples operaciones interdependientes: selección de la información relevante; organización y vinculación de los datos; y definición del medio y el lenguaje a utilizar, ya que la forma de representación que se elige restringe lo que se es capaz de decir, simplemente algunos aspectos de la experiencia humana se expresan mejor en ciertas formas que en otras. Así, la elección de una forma de representación equivale a una manera de concebir el mundo (Eisner [1998], en Muñoz, 2000).

Según Chiarella (2009), los avances en el campo representacional sucedidos a lo largo de la historia, repercutieron en las maneras de concebir el espacio. Los cambios en los sistemas de representación provocaron modificaciones sustanciales en los procesos de proyectación y por ende, en las concepciones espaciales. El paradigma de la complejidad, que nace del cuestionamiento de los métodos y teorías del modelo científico positivista, migra desde rígidas verdades universales hacia múltiples miradas en permanente reformulación. La exploración gráfica sugiere nuevos caminos a partir de la resignificación de los tradicionales sistemas de representación y de la incorporación de recursos innovadores de simulación y construcción digital.

Con el surgimiento de interfaces naturales y ubicuas, a través de las cuales los datos se adhieren a los objetos del entorno cotidiano o acompañan a los sujetos cualquiera sea su ubicación geográfica, el desafío es transformar los datos en conocimientos, mediante representaciones capaces de articular principios éticos y estéticos con nuevas posibilidades de experiencia.

Este complejo escenario es conformado y experimentado como una red de procesos interconectados en múltiples niveles de articulación, que entrecruza su telar en un espaciotiempo multidimensional, elástico y en constante expansión, convertido en un flujo continuo que impone su espacialidad neobarroca, topológica e infinita.

\section{Agradecimientos}

Al Instituto Rosario de Investigaciones en Ciencias de la Educación (CONICET-UNR) por facilitar los datos para el estudio de caso, principalmente a su vicedirectora la Dra. Patricia San Martín; y a la Secretaría de Ciencia y Técnica de la Universidad Nacional del Litoral por hacer posible la participación en este congreso.

\section{Referencias}

Alexander, C. (1965). La ciudad no es un árbol. Berkeley: Universidad de Berkeley.

Chiarella, M. (2009). Unfolding Architecture. Laboratorio de Representación e Ideación. Tesis de doctorado. Barcelona: Universidad Politécnica de Catalunya.

Doberti, R. (1998). Habitar el Desasosiego. Recuperado de https://docs.google.com/file/d/OBxH72auEtxNwMWEyZG MzYjMtYzEzMy00MzUzLTg5NzQtYTc3ZWY3ZTA5ZWVj/ edit?hl=en\&pli=1 
Heidegger, M. (2001) [1954]. Conferencias y ensayos. Barcelona: Editorial Delserbal.

Levy, P., Authier, M. (1996) [1992] Los árboles de conocimientos. París: La découverte.

Manovich, L. (2013). Para entender los metamedios. En Software takes comand. Bloomsbury Academic. Publicado bajo licencia Creative Commons en manovich.net. Traducción de Reyes-García. Recuperado de: http://manovich.net Maturana, H., Varela, F.(2003)[1984]. Elárbol del conocimiento: las bases biológicas del conocimiento humano. Buenos Aires: Lumen.

Muñoz, P. (2000). Diversidad e Igualación: Informática y
Material Didáctico para la Enseñanza de Morfología en Diseño. En: Construyendo el espacio digital. Río de Janeiro: Sigradi 2000. Recuperado de http://cumincad.scix.net/data/ works/att/6472.content.pdf

Rancière, J. (2010). El Espectador Emancipado. Buenos Aires: Manantial.

Rodríguez, G. (2011). La teoría de los sistemas complejos aplicada al modelado del dispositivo hipermedial dinámico. Tesis de doctorado. Facultad de Ciencias Exactas, Ingeniería y Agrimensura, Universidad Nacional de Rosario. Recuperado de http://rephip.unr.edu.ar/handle/2133/4226 